\title{
GPPS-BJ-2019-0016
}

\section{Modelling and analysis the thin wall film formation with integrated discrete and continuous phase simulation approach}

\author{
Peng KE, Jie LIU, Yi LU \\ School of Transportation Science and Engineering, Beihang University \\ p.ke@buaa.edu.cn \\ Beijing, 100191, China
}

\begin{abstract}
Water film will be initiated and formed under the continuous impingement of incoming supercooled droplets, and directly affect the ice formation, which is always vital for the performance and safety of aero-engine. A hybrid algorithm integrating Discrete Phase Model (DPM) and Volume of Fluid (VOF) was established to simulate all the stages from droplet initiation to the film well-formed which can make the numerical simulation of icing more realistic and accurate. The transformation criterion from the particle to liquid volume fraction and the distribution of source terms has been improved compared with similar previous approaches. Three verification cases, namely single droplet train impact, staggered droplets impact and two glycerine-solution droplet impact, were conducted to verify the mass conservation and the applicability to both the sparse and dense two-phase flow. The results showed that the relative error of mass transformation is no more than $0.5 \%$. The comparison between simulation and experiment result demonstrated that the hybrid algorithm has the ability to simulate the flow behaviour of droplets accurately. The thin water film formation on a typical wing of NACA0012 airfoil was then analysed, the results of which illustrated that the integrated algorithm could successfully capture the characteristics of liquid film formation and motion.
\end{abstract}

\section{INTRODUCTION}

Liquid film will form under the continuous impingement of droplets in many industry fields, such as ice accretion process (Rothmayer et al., 2002) (Moore, et al.,2017) on the engine inlet vane or nosecone, fuel spray process inside combustion chamber (Pan et al., 2019), or thin liquid films on steam turbine airfoils (Rossi et al., 2017). It has very important influences on the system operation and flight safety.

Icing is always vital for the performance and safety of aero-engine, during the icing process inside aero-engine, the water film will be initiated and formed under the continuous impingement of incoming supercooled droplets, and then flow under the shear force from the mainstream. Thus, calculating the flow pattern accurately after the droplet impingement is of great significance as well as the basis of subsequent ice shape calculation.

The formation of wall film during the impingement process has been modelled and investigated with different methods, although the classic Messinger model for icing simulation does not consider the water film flow on the ice layer. Some researches (Myers and Charpin, 2004) (Nakakita et al., 2010) (Cao et al., 2016) (Chauvin et al., 2018) adopted the lubrication equation based on the assumption of continuous and thin liquid film to model the film height, which is validated for the phase where the film has formed well and is unable to consider the initial icing roughness, which has great influence on calculating the shape of ice accurately.

The global "equivalent sand grain roughness" whose height does not change with time and space was used to simulate effects of the surface roughness on the flow and heat transfer process. However, it still cannot reproduce the different size of ice beads observed in experiments. In the meantime, beyond the droplet impingement limits, broken of continuous liquid film will happen due to icing or evaporation. Therefore, some other models must be developed to simulate the formation of rivulet and initial roughness, such as the work initiated by Guy (Guy Fortin, et al., 2006) and followed by Li (Li, et al., 2011) and Chang (Chang, et al., 2014), where the roughness prediction model was established after analysing the forced characteristics of water for three different forms of flow, namely water droplets, liquid film and stream.

The VOF approach in Eulerian framework could perfectly simulate the liquid water distribution, but the computational cost is extremely high. To balance the requirements of computation capacity and accuracy, a coupled method combined DPM and VOF is brought out to simulate the flow behaviour of super-cooled large droplets after impingement, which has shown some prospects in the field of two-phase flow simulation.

Chou et.al (Chou et al. 2015) presented a two-way coupled Eulerian-Lagrangian model to simulate the suspension of fine particles in liquid flows, which demonstrated that the integrated model is capable of revealing the detailed features of particle-laden flows. Zuzio et.al (Zuzio 
et.al, 2018) developed an improved Eulerian-Lagrangian coupled approach to ensure a smooth transition between each modelling frameworks in the zones where they reach their respective limit s of validity, in which the ability of the method to reproduce the whole atomization process was highlighted. Anez et.al (Anez et al., 2019) focused on the unresolved interface and tested the model against realistic experimental data, where indicated that models that are computationally less demanding and still accurate enough are required in an industrial environment. Maeda et.al (Maeda et al., 2018) presented a coupled Eulerian-Lagrangian method to simulate cloud cavitation in a compressible liquid for capturing the strong oscillations of each bubble. All these studies show the efficiency and promising application of the integrated discrete and continuous phase simulation approach.

Recently, some researchers have extended such models to investigate the process of water film formation. Arienti et.al (Arienti et al. 2010) proposed a computationally tractable model for film formation and breakup, where the film was modelled using VOF technique and droplets formed after film breakup were tracked by the parcel approach. Adeniyi et.al (Adeniyi et al., 2017) proposed an enhanced VOF technique to model the oil and gas flow inside an aero-engine bearing chamber, where the droplets were tracked in a Lagrangian framework and coupled to the Eulerian phases.

An integrated discrete and continuous phase simulation approach extended from reference was established in this study to simulated all the stages from initiation to the film well formed to give a better start pointing to the later simulation of icing. Firstly, the physical problem and the overall framework of the DPM-VOF coupled algorithm were theoretically defined, and the governing equations of the models used were given. Secondly, the improvements in two aspects of transformation criterion and reallocation of source terms were compared with precious similar algorithm. Then the improved algorithm was verified by three cases, namely impact of a droplet train, impact of staggered trains and impact of two large droplets, respectively. At last, the coupled algorithm was used to simulate the initial stage of liquid film formation on NACA 0012 airfoil and the corresponding analysis was given.

\section{METHODOLOGY AND VERIFICATION}

When aircraft flies through clouds with super-cooled liquid droplets, as described in FAR Part 25 and Part 33, droplets tend to impact the upstream-facing surfaces on the aircraft or engine. The droplets freeze completely immediately upon impact when the ambient air temperature is far below the freezing point. However, the latent heat of fusion will be released from the freezing water and warm the accreted ice to form thin wall film when there is high liquid water content (LWC) and/or air temperatures only slightly below the freezing point. The unfrozen water film tends to run back along the surface under the shear force from outside airflow and may eventually freeze due to the continuous convective heat transfer to the ambient cold air.

According to the icing process mentioned above, it can be found that, the whole flow domain could be divided into two regions, the far field region, where only droplets move, and the near wall region, where ice accretes and film flows. The film flow and ice accretion process are demonstrated in Fig. 1.

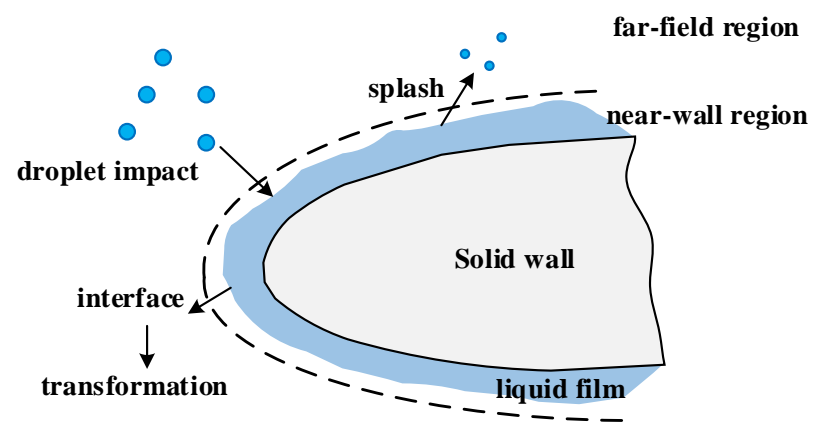

Fig. 1 Film flow and ice accretion

The theories of this algorithm, including the overall framework, DPM model, the mass, momentum and energy conversion models are introduced in detail.

\section{Algorithm framework}

A hybrid algorithm integrating DPM and VOF was established to simulate all stages from droplet initiation to the well-formed film to make the numerical simulation of icing more realistic and accurate.

For the droplet (the dispersed phase), once it is injected into the flow field, its trajectory is calculated in Lagrangian form and updated at each time step of the unsteady flow calculation. When it impinges on the dry wall or wall film, its mass, momentum and energy will transfer into the water film. Then, the water film (the continuous phase) will be modelled using VOF method, where the advection equation for the liquid volume fraction is formulated in volume of fluid form using the compressive interface capturing scheme for arbitrary meshes (CICSAM) scheme. The multiphase calculation is carried out with FLUENT's pressure-based solver using the implicit body force treatment to account for the surface tension term.

\section{Governing equations for discrete phase}

Considering the smaller volume of fraction and larger density of droplets in icing conditions, several assumptions are made as follows,

(a) The droplets are spherical without deformation, breaking, collision, and coalescence,

(b) The droplets will stick without rebounding when hitting on a solid wall or thin film,

(c) The mass transfer between the droplets and the surrounding air flow are ignored.

The equations of motion of the droplet can be written as:

$$
\begin{gathered}
\frac{\mathrm{d} \mathbf{U}_{p}}{\mathrm{~d} t}=\frac{18 \mu}{\rho_{\mathrm{d}} d^{2}} \frac{C_{D} R e}{24}\left(\mathbf{u}-\mathbf{U}_{p}\right)+\frac{\rho_{\mathrm{d}}-\rho}{\rho_{\mathrm{d}}} \mathbf{g} \\
\operatorname{Re}=\frac{\rho_{l}}{\mu} D_{p}\left|\mathbf{U}_{\mathbf{p}}-\mathbf{u}\right|
\end{gathered}
$$




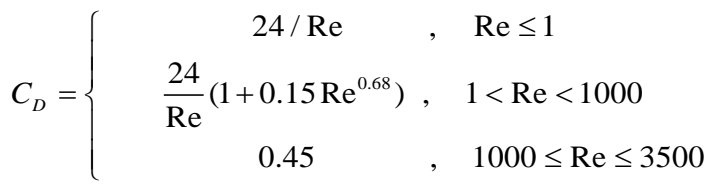

The energy equation for the droplet is,

$$
m_{p} c_{p} \frac{d T_{p}}{d t}=h A_{p}\left(T_{\infty}-T_{p}\right)+\frac{d m_{p}}{d t} h_{f g}
$$

\section{Governing equations for continuous phase}

The continuity equation considering the volume of fraction for each phase is,

$$
\begin{aligned}
& \frac{\partial\left(\alpha_{i} \rho_{i}\right)}{\partial t}+\nabla \cdot\left(\alpha_{i} \rho_{i} \mathbf{u}\right)=S_{\rho} \\
& S_{\rho}=\frac{\rho_{i}}{\rho_{l}} \frac{M_{p}}{\Delta t} \frac{1}{V_{\text {cell }}} \delta \\
& \delta=\left\{\begin{array}{l}
+1, \text { for water } \\
-1, \text { for air }
\end{array}\right.
\end{aligned}
$$

$\delta$ is a switching function, which can make a judgement correspondingly to bring the source term into force when approaching the wall or liquid film.

The monument equation is given in Eq. (8). This represents the force balance at each point in the domain. The inertial terms are balanced with the divergence of stress, body forces and other contributory source terms.

$$
\frac{\partial}{\partial t}(\rho \mathbf{u})+\nabla \cdot(\rho \mathbf{u u})=-\nabla p+\nabla \mu\left[\nabla \mathbf{u}+\nabla \mathbf{u}^{T}\right]+\mathbf{F}+\mathbf{S}_{\mathbf{m}}
$$

where $\mathbf{F}$ is the total body forces, including the surface tension force transformed using the continuum surface force (CSF) model proposed by Brackbill et al. (Brackbill et al., 1992), which interprets surface tension as a continuous, threedimensional effect across an interface, rather than as a boundary value condition on the interface. $\mathbf{S}_{\mathrm{m}}$ is the source term caused by the impacted droplet and calculated as,

$$
\mathbf{S}_{\mathbf{m}}=S_{\rho} \mathbf{U}_{\mathbf{p}} \delta
$$

The energy equation is given in Eq. (10), where the energy and temperature are averaged from each phase, as in Eq. (11) and (12).

$$
\begin{gathered}
\frac{\partial}{\partial t}(\rho E)+\nabla \cdot(\boldsymbol{u}(\rho E+p))=\nabla \cdot\left(k_{e f f} \nabla T\right)+S_{t} \\
E=\frac{\sum_{i=1}^{n} \alpha_{i} \rho_{i} E_{i}}{\sum_{i=1}^{n} \alpha_{i} \rho_{i}} \\
T=\frac{\sum_{i=1}^{n} \alpha_{i} \rho_{i} T_{i}}{\sum_{i=1}^{n} \alpha_{i} \rho_{i}}
\end{gathered}
$$

The droplet is assumed to mix adiabatically and instantly when it impacts on the film, as calculated in Eq. (13).

$$
T_{i}=\frac{M_{f} T_{f}+M_{p} T_{p}}{M_{f}+M_{p}}
$$

\section{Implementation}

Compared with previous integrated discrete and continuous phases simulation methods presented by other researchers, this coupled approach has been improved in the following two aspects.

\section{Transformation criterion}

The transformation from droplet into film needs to be activated as soon as the particle impacts the wall or the free phase interface. In previous researches, the criterion of transformation uses the centre position of the droplet $\left(P_{0}\right)$ to judge the relative relationship between the particle and the interface, which leads to transformation delay because the bottom half of the particle has already immerged into the interface.

In this study, as is shown in Fig.2, $P_{1}$ is the lowerboundary point of the spherical droplet along its velocity $\left(\mathbf{U}_{\mathbf{p}}\right.$ ) direction. The criterion for initiating the transformation is thus improved, using $P_{1}$ to judge the relative position between the interface and the droplet needed to be converted.

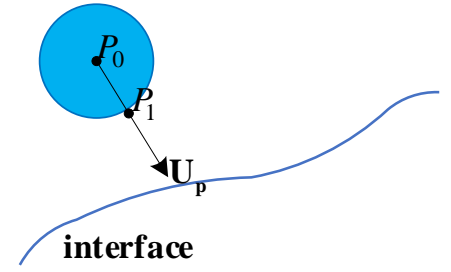

\section{Fig. 2 Relative position of judgement}

Cells are searched layer by layer from the wall boundary along the normal direction until the liquid volume fraction in the current cell is less than the critical value (defined as 0.35). These cells will be marked as the interface of gas-liquid phases, of which the corresponding liquid film thickness will be stored. When $P_{1}$ enters the marked region, transformation will be activated. The main logic of the implementation is shown in Fig.3.

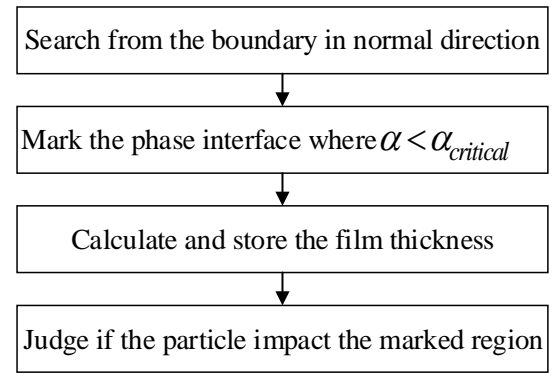

Fig. 3 DPM-VOF transformation

\section{Reallocation of source terms}

Then the mass and momentum of the incoming droplet will be reallocated into the cells in terms of liquid volume fraction, as the source terms in Eq. (5) and (8). The initial 
value of such reallocation of liquid volume fraction will affect the iterative efficiency of subsequent VOF process.

The search scope $R_{s}$ is defined here as the radius of the spherical droplet extending out half of the maximum distance of the cell in three directions, taking the centre of the droplet as the centre of searching, which can be expressed as Eq. (14) and demonstrated in Fig. 4.

$$
R_{s}=0.5 \cdot D_{p}+0.5 \cdot \max \{d x, d y, d z\}
$$

Furthermore, all cells will be marked and the mass will also be added into each one according to the relative distance between it and the droplet,

(1) Marked as "1", where the cell is partly inside the search scope. Mass will be added by weight according to the distance $R_{i}$ between the cell centre and droplet centre, as in Eq. (15).

$$
m_{1 i}=\left(\frac{1}{R_{i}}\right) /\left(\sum_{i=1}^{n} \frac{1}{R_{i}}\right)
$$

(2) Marked as " 2 ", where the cell is totally inside the search scope. The mass will be added into such cells according to the mass source item and the volume of the current cell.

The total mass added into all cells of these two kinds should satisfy the mass conservation relationship during transformation. All the other cells completely outside the search scope will be marked as " 0 ", into which mass will not be added into.

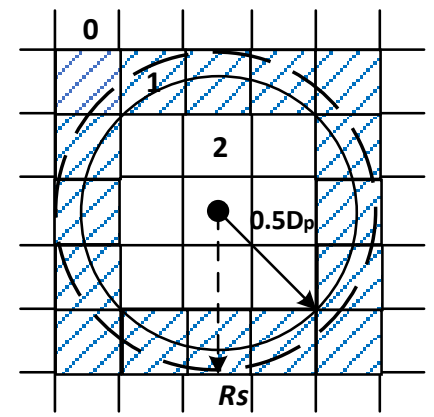

Fig. 4 Demonstration of search scope and cell

$$
\text { marking in 2D }
$$

A sample of a marked droplet is shown in Fig. 5.

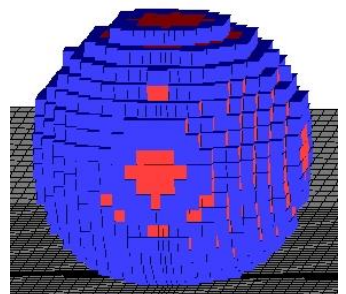

Fig. 5 Sample of marked drop in 3D (1:blue, 2: red)

\section{Verification}

Some verification simulations of droplets impingement were conducted to verify that the hybrid algorithm is effective and applicable to both the sparse and dense two-phase flow, especially the mass conservation during transformation from
DPM into VOF and the flow behaviour after the transformation.

Compared with the Eulerian-Eulerian algorithm in high precision, the integrated algorithm was not demanding on the quality of the grid. To be more specific, the droplet in the far field region is treated as spherical particles whose shape does not change with motion. It is only necessary to densify the mesh in the near-wall region where the transformation occurs to obtain a well capture of the gas-liquid phases interface. And the total calculation time could be shortened by at least one order of magnitude. The time step is limit to $10^{-6}$ to keep the Courant number less than 1 . The convergence criteria set for all the equations is $10^{-4}$.

\section{Case one: impact of a droplet train}

The first case is a water droplet train impact onto a flat plate, as shown in Fig. 6, where the droplet diameter is $0.5 \mathrm{~mm}$, initial velocity is $1 \mathrm{~m} / \mathrm{s}$, height is $2 \mathrm{~mm}$, flux is $0.7854 \mathrm{~g} / \mathrm{s}$, contact angle between the droplet and wall is settled as $150^{\circ}$ to simulate the hydrophobic surface.

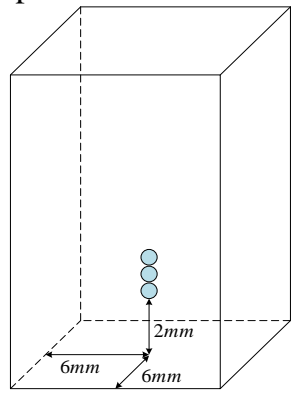

Fig. 6 water droplet train impact onto a flat plate

The successful conversion of discrete droplets into continuous phase is demonstrated in Fig. 7, where the contour is obtained by taking the equivalent surface of $\alpha=0.5$ in the liquid phase volume as the interface between air and liquid phases. The growth and deformation process of large droplets which converge on the wall of $0.0098 \mathrm{~s}, 0.02 \mathrm{~s}$ and $0.2 \mathrm{~s}$ are shown in Fig. 7 a), b), c) respectively. Because the discrete small droplet falls down to the wall surface with a certain speed, so the conversion to the continuous phase will first expand along the wall under the drive of the inertial force, and then quickly shrink under the action of surface tension, reciprocating several cycles before stabilization. Because the wall static contact angle is set as $150^{\circ}$, a hemispheric body with good symmetry is formed after $0.2 \mathrm{~s}$, showing the shape of droplet surface, which is in good agreement with the actual condition.

Mass conservation check was conducted after $0.2 \mathrm{~s}$, where the total mass of injected droplets was summed to $0.7854 \mathrm{~g} / \mathrm{s}$ $\times 0.2 \mathrm{~s}=0.1571 \mathrm{~g}$. All the water transformed inside the cell whose VOF is bigger than $10^{-6}$ is $0.1564 \mathrm{~g}$, of which the error is no more than $0.43 \%$. 


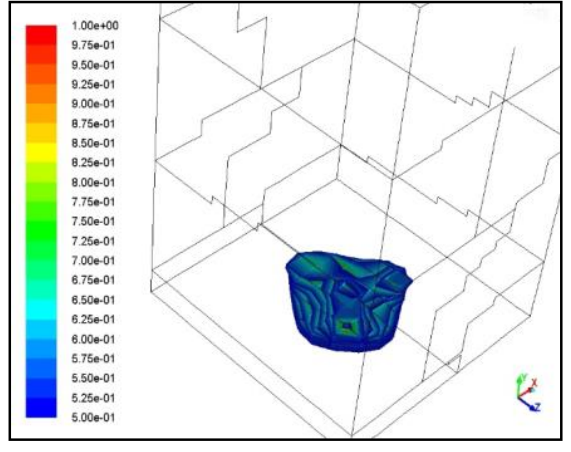

a) $0.0098 \mathrm{~s}$

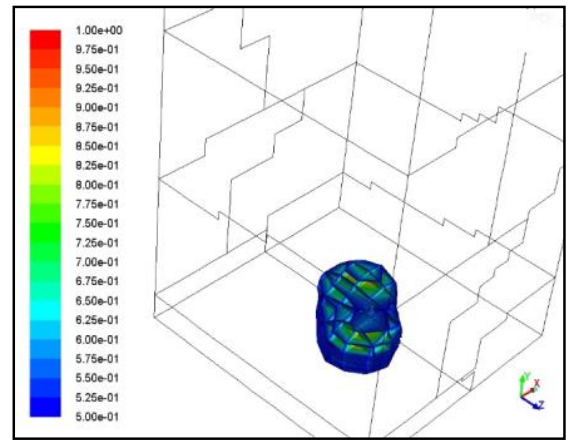

b) $0.02 \mathrm{~s}$

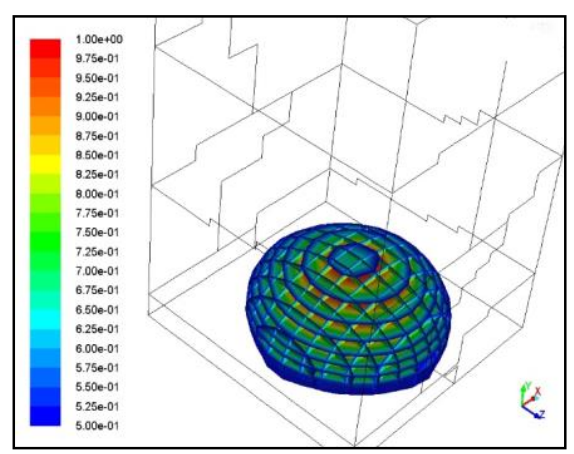

c) $0.2 \mathrm{~s}$

Fig. 7 Transformations after impact

\section{Case two: impact of staggered droplet trains}

More droplets arranged in a surface with staggered grid were injected to impact the flat plate in this case, as shown in Fig. 8, Fig. 9 are the characteristic forms of droplets under wall effects at different time, which fully show the interaction between droplets and wall surfaces. The droplet diameter is $0.25 \mathrm{~mm}$ with a total mass flow rate of $3.1 \mathrm{~g} / \mathrm{s}$. The static contact angle between wall and the droplet is set as $150^{\circ}$ to simulate the hydrophobic surface. As can be seen in Fig. 9, due to the influence of wall surfaces, the droplets at four corners will first occupy a larger area, and then form an approximate eight-part sphere under the action of surface tension. Next, the large droplets in the four corners will continue to grow under the impact of a steady stream of discrete droplets, contacted and gradually combined with the nearest droplet at $0.047 \mathrm{~s}$.

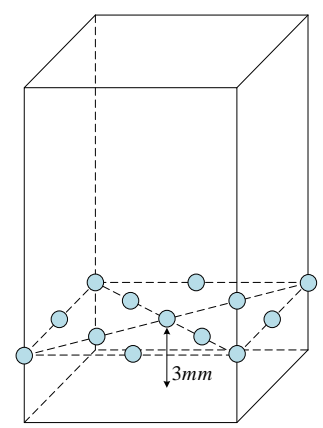

Fig. 8 staggered droplets impact on a flat plat

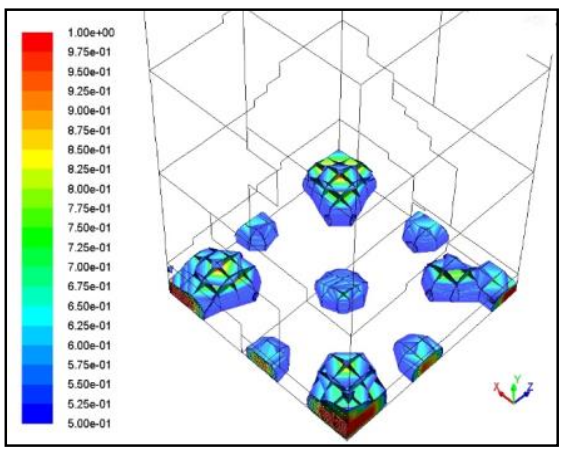

a) $0.023 \mathrm{~s}$

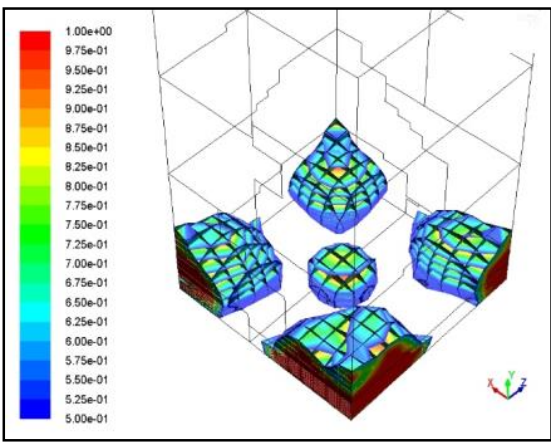

b) $0.047 \mathrm{~s}$

Fig. 9 Transformations after impact

Mass conservation check was then conducted after $0.047 \mathrm{~s}$, where the droplets injected was summed to $3.1 \mathrm{~g} / \mathrm{s} \times$ $0.047 \mathrm{~s}=0.1457 \mathrm{~g}$. All the water transformed inside the cell whose VOF is bigger than $10^{-6}$ is $0.1455 \mathrm{~g}$, of which the error is no more than $0.14 \%$.

\section{Case three: impact of two large drops}

$87 \%$ glycerine-in-water solution droplets impacting on a flat plate were simulated and compared with the experiment done by Dalili (Dalili et al., 2012). As shown in Fig.10 a), the first droplet was injected $3 \mathrm{~mm}$ above the centre of the bottom plate. The diameter and the impacting velocity were $3.4 \mathrm{~mm}$ and $1.1 \mathrm{~m} / \mathrm{s}$, respectively. The stable shape was obtained under the condition that the wall static contact angle is $60^{\circ}$, as illustrated in Fig.10 b). The mass of the converted droplet is $2.542 \mathrm{e}^{-5} \mathrm{~kg}$, the relative error is less than $0.489 \%$ given the mass of the injected particle is $2.529 \mathrm{e}^{-5} \mathrm{~kg}$. 


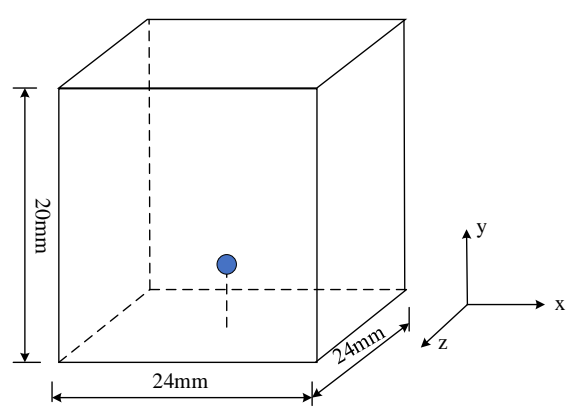

a) schematic of injection

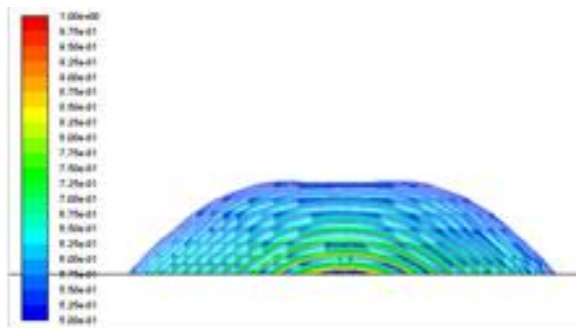

b) stable shape of the first droplet

\section{Fig. 10 First glycerin droplet impact on a flat plane}

The second droplet was then injected at a distance of $0.73 \mathrm{~mm}$ shifted in $\mathrm{x}$ direction from the first one, the flow behaviour after contact was recorded and compared with the experiment results. It can be seen from Fig. 11 that at $0 \mathrm{~ms}$, the lower surface of the second droplet just comes into contact with the upper edge of the first one, and both of them maintain their own original shape. As the second drop falls and sinks into the first one, a slanted, bulging contact line appears, and gradually spread around, thus forming a circular table with a lower left side and a higher right side. After $10 \mathrm{~ms}$, the two droplets completely merged to form a pancake. All of the aforementioned details are well captured by the DPM-VOF coupled algorithm used in this paper as well as match well with the experimental results. Thus, a conclusion can be drawn that the integrated algorithm is enough accurate to simulate the droplet growth and deformation

\section{FILM INITIATION AND FORMATION ON WING}

The thin water film formation on a typical wing with NACA0012 airfoil, in Fig. 12, was then analysed with the integrated discrete and continuous phase simulation approach. Results illustrated that the integrated algorithm could successfully capture the characteristics of liquid film formation and motion. For instance, since the water droplets in the incoming flow impact onto the front of the structure along $\mathrm{x}$-axis direction, a large part of water accumulates in the leading edge region, and the "single film peak" occurs at about $0.15 \mathrm{~m}$ from the leading edge of the airfoil. Then the liquid film ruptures into a multi-rivulet distribution, which slowly flows back under the action of airflow. Due to the K-H instability. The surface of the liquid film will fluctuate to some extent.

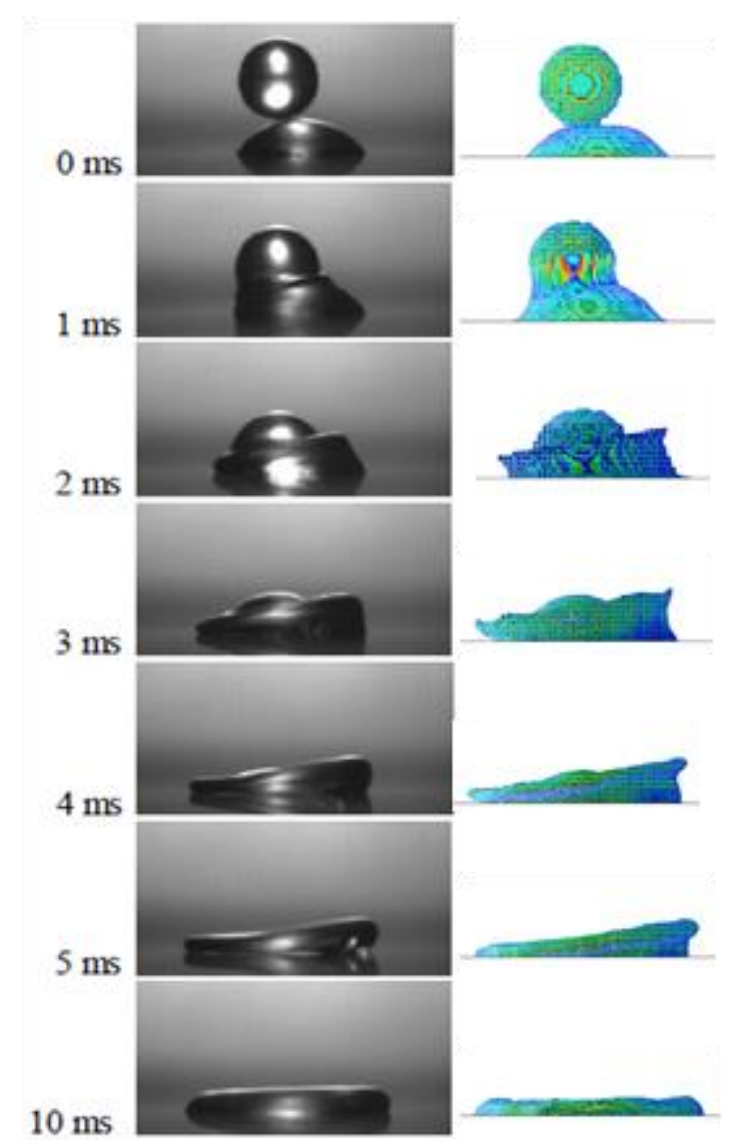

(a) Reference experiment

(b) Our simulation Fig. 11 Flow behavior after impact
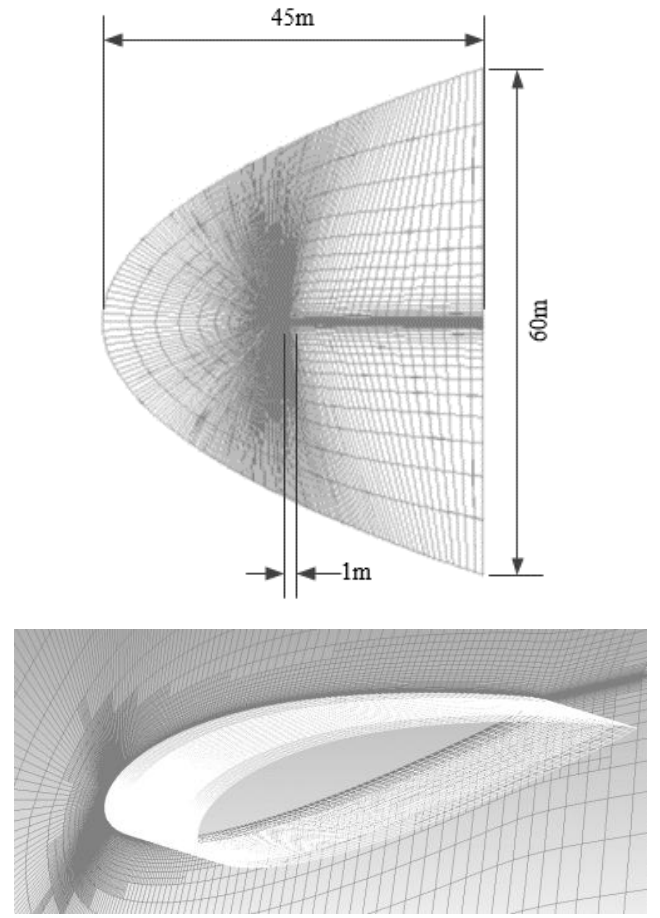

Fig. 12 Computational domain and mesh

The wall contact angle was set as 90 degree, and time step for transit simulation is $10^{-4} \mathrm{~S}$ 。 


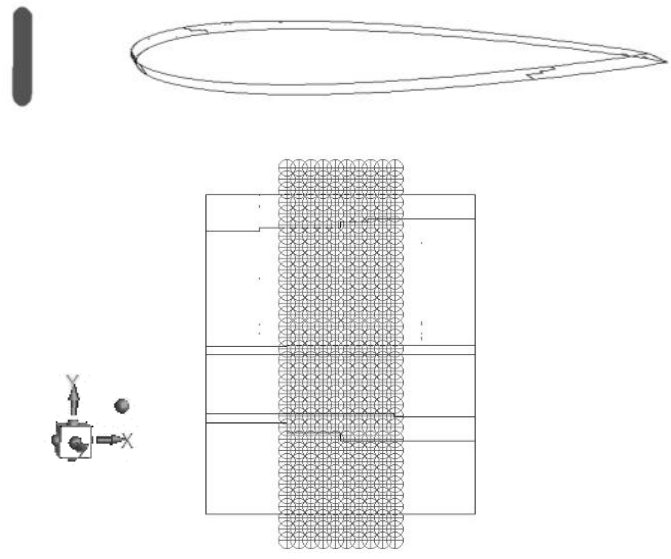

Fig. 13 Initial release position and spatial arrangement droplet.

The initial release positions and spatial arrangement of a batch of particle groups in front are shown in Fig. 13, assuming that the droplet swarm moves continuously to the airfoil in the form of an array from the front edge of the wing $0.2 \mathrm{~m}$ ( $\mathrm{x}$ direction), the initial distribution range in $\mathrm{y}$ direction is $-0.07 \mathrm{~m} \sim 0.07 \mathrm{~m}$, and in $\mathrm{z}$ direction is $0.03 \mathrm{~m} \sim 0.07 \mathrm{~m}$. The droplet arrays are arranged as $34 \times 10$. The droplets have a uniform diameter of $0.2 \mathrm{~mm}$, the speed are set as $20 \mathrm{~m} / \mathrm{s}$ along the $\mathrm{x}$ direction. Because the physical quantity affecting the initial liquid film state is in a certain functional relationship with the product of space liquid water content (LWC) and time $(\mathrm{t})$, the calculation can shorten the time of transient numerical calculation by appropriately increasing LWC. A dimensionless time $t^{*}$ is defined as Eq. (16).

$$
t^{*}=\frac{E_{m} \cdot L W C \cdot \boldsymbol{u}}{\rho \cdot L} \cdot t(16)
$$

$E_{m}=1$ is the total water collection rate, $L W C$ equals to $10 \mathrm{~kg} / \mathrm{m}^{3}$ in this case, $L$ is the characteristic length, equals to the chord length $1 \mathrm{~m}$ in this study.

In this case, the total flow rate of droplets was set as $0.11373 \mathrm{~kg} / \mathrm{s}$, whose diameter were $0.0002 \mathrm{~m}$ with the velocity of $20 \mathrm{~m} / \mathrm{s}$. The droplets were released in the form of particles, the entire trajectory of which is shown in Fig. 14, the color shades represent the time of motion of the particles.

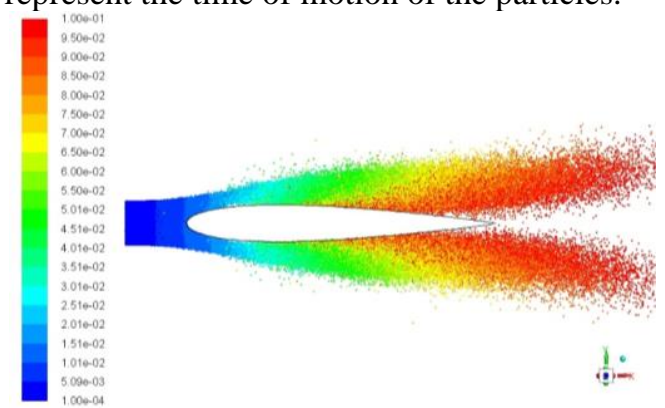

Fig. 14 Trajectories of droplets

Taking the contour of volume of liquid $\alpha=0.5$ as the interface between air and liquid phases, Fig. 15 shows the contour boundary of the wing surface and the liquid phase in the space at $t^{*}=0.004008$ in the transient process.

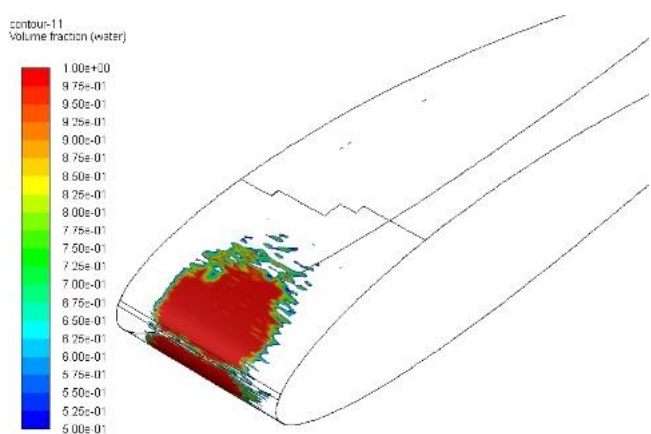

a) On the wing surface

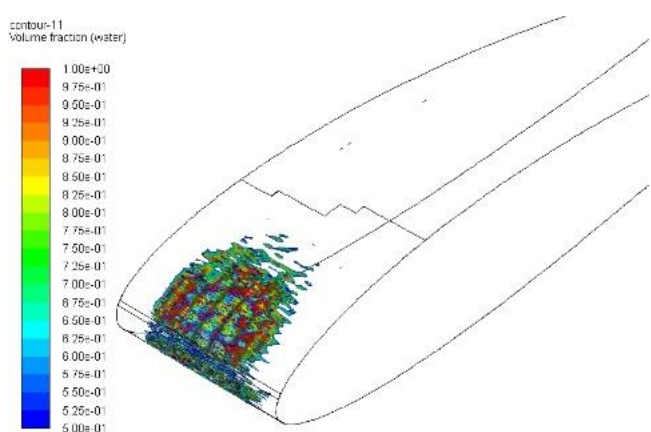

b) In 3D space

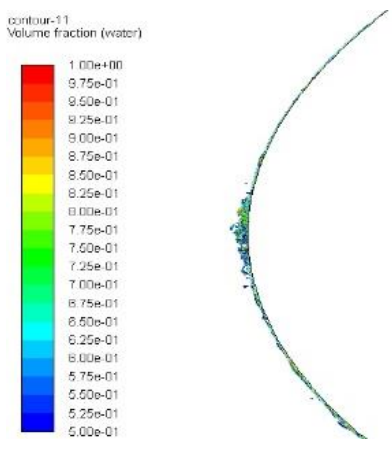

c) In 3D space (side view)

Fig. 15 The VOF of liquid phase

The thickness variation of the liquid film in chord wise is further analyzed, and three lines distributed equally along the wing surface are taken as the characteristic location, as shown in Fig. 16, where the locations at $\mathrm{z}=0.025 \mathrm{~m}$ and $0.072 \mathrm{~m}$ are outside of the region of directly impingement and the film formed by the flooding water from impingement limits.

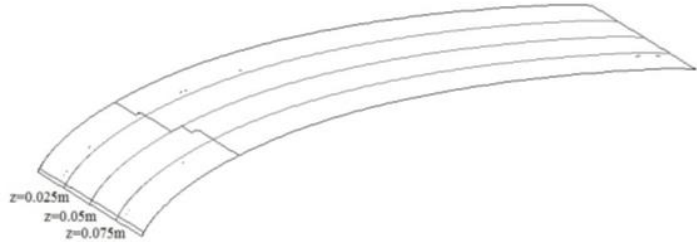

\section{Fig. 16 Characteristic location on the surface}

The liquid film thickness on each characteristic location at different times are draw out respectively, which is shown in Fig. 17. It can be seen that the variation trend of film thickness on the feature lines is similar, and the film on the location of $0.025 \mathrm{~m}$ are thinner than that of $0.05 \mathrm{~m}$. 
The changes of the liquid film thickness along the chord wise at different times on different characteristic lines were observed, and the curves were similar too. The liquid film formed from droplets impacting the wing surface are flattening from a combination of their initial momentum and the shear force imposed by the air stream at the initial stage of $t^{*}=0.004008$. After then, with the rapid and continuous impingement of droplets later and relative slowly movement of film due to the wall friction and surface tension at front head, more water accumulates not far from the stagnation point and forms some peaks, which grows higher and moves downward due to the impact momentum and shear force from air. The difference between peak and valley are also getting larger, the average movement speed of the peak is $1.6 \mathrm{~m} / \mathrm{s}$.

Besides that, the ripple, which is the evolution of interfacial waves on thin liquid films driven by an air boundary layer, appears when $t^{*}=0.014028$, slowly moves down ward and grow higher too.

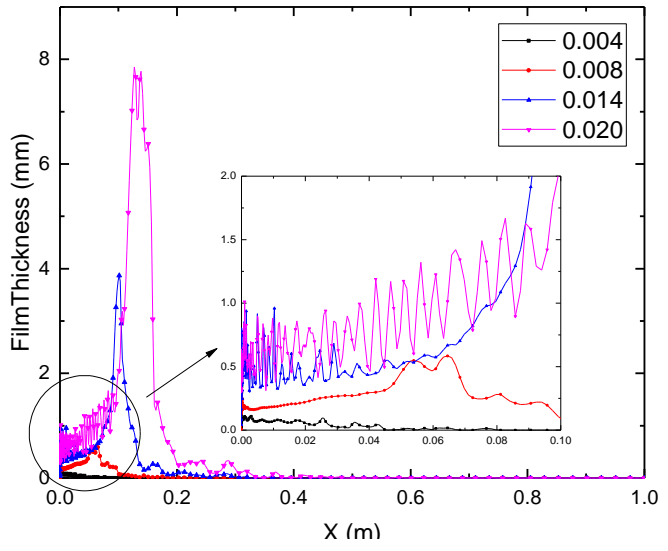

a) $z=0.025 \mathrm{~m}$

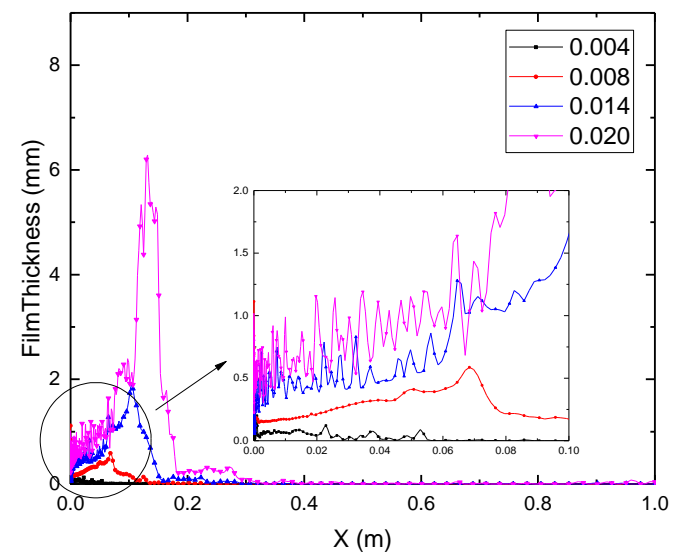

b) $z=0.05 m$

Fig. 17 Liquid Film thickness at different time

\section{CONCLUSION}

The integrated algorithm are brought out and validated, it successfully captures some details of the formation and movement of the liquid film on the wing of NACA0012 airfoil, such as the movement of the liquid film along the chord wise and the moving, the "single peak" of the liquid film, the "stream" after the film and the ripple phenomenon caused by the instability of the liquid film plane due to K-H instability .

To be noted, the influence of static contact angle and flow velocity will have great effects on the shape of the liquid film and will be analysed in the future.

\section{REFERENCE}

1) Adeniyi A., Morvan H., and Simmons, K. (2017). A coupled Euler-Lagrange CFD modelling of droplets-tofilm. The Aeronautical Journal 121(1246), 1897-1918.

2) Anez J., Ahmed A., Hecht N., Duret B., Reveillon J., and Demoulin F. X. (2019). Eulerian-Lagrange Spray Atomization Model Coupled with Interface Capturing Method for Diesel Injectors. International Journal of Multiphase Flow (in press).

doi:10.1016/j.ijmultiphaseflow.2018.10.009

3) Arienti M. M., Wang L. L., Corn M. M., et al. (2010). Modeling Wall Film Formation and Breakup Using an Integrated Interface-Tracking/ Discrete-Phase Approach. ASME. Turbo Expo: Power for Land, Sea, and Air, Volume 2: Combustion, Fuels and Emissions, Parts A and B ,1059-1068.

4) Brackbill J. U., Kothe D. B., and Zemach C. (1992). A Continuum Method for Modeling Surface Tension. Comput. Phys.100. 335-354.

5) Chang S. N., Wang C., Zhao Y. Y., et al. (2014). Modeling of Roughness Dimension and Distribution on the Icing Surface. ACTA Aerodynamic SINICA, 32 (5), 660-667.

6) Cao Y. H., and Hou S. (2016). Extension to the Myers Model for Calculation of Three-Dimensional Glaze Icing, Journal of Aircraft, 53 (1), 106-116.

7) Chauvin R., Bennani L., Trontin P., and. Villedieu P. (2018). An implicit time marching Galerkin method for the simulation of icing phenomena with a triple layer model, Finite Elements in Analysis and Design 150, 20 33.

8) Chou Y. J., Gu S. H., Shao Y. C. (2015). An EulerLagrange Model for Simulating Fine Particle Suspension in Liquid Flows. Journal of Computational Physics 299, 955-973.

9) Dalili A., Chandra S., Mostaghimi J., et al. (2012) Formation of Liquid Sheets by Deposition of Droplets on a Surface. ILASS America: 24th Annual Conference on Liquid Atomization and Spray Systems, 1-8.

10) Fortin G., Laforte J. L., and Ilinca A. (2006). Heating and Mass Transfer during Ice Accretion on Aircraft Wings with an Improved Roughness Model. International Journal of Thermal Sciences, 45, 595-606.

11) Li Y., Wang C., Chang S. N., et al. (2011). Simulation of Ice Accretion based on Roughness Distribution. Procedia Engineering, 17, 160-177.

12) Maeda K., Colonius T. (2018). Eulerian-Lagrangian Method for Simulation of Cloud Cavitation. Journal of Computational Physics 371, 994-1017.

13) Moore M. R., Mughal M. S. and Papageorgiou D. T. (2017). Ice formation within a thin film flowing over a flat plate, J. Fluid Mech 817, 455-489.

14) Myers, T. G., and Charpin J. P. F. (2004). A Mathematical Model for Atmospheric Ice Accretion and Water Flow on 
a Cold Surface. International Journal of Heat and Mass Transfer 47 (25), 5483-5500.

15) Nakakita K., Nadarajah S., and Habashi W. (2010) Toward Real-Time Aero-Icing Simulation of Complete Aircraft via FENSAP-ICE, Journal of Aircraft 47 (1), 96-109.

16) Pan H. J., Xiao D, Hunga D., Xu M., et al, (2019). Experimental investigations of wall jet droplet impact on spray impingement fuel film formation, Fuel 241, 33-41.

17) Rossi P., Raheem A., and Abhari R. S. (2017). Numerical Model of Liquid Film Formation and Breakup in Last Stage of a Low-Pressure Steam Turbine. ASME. J. Eng. Gas Turbines Power.;140 (3).

18) Rothmayer A.P., Matheis B.D., and Timoshin S.N. (2002), Thin liquid films flowing over external aerodynamic surfaces, Journal of Engineering Mathematics 42, 341-357.

19) Zuzio D., Estivalezes J. L., DiPierro B. (2018). An Improved Multiscale Eulerian-Lagrange Method for Simulation of Atomization Process. Computers and Fluids 176, 285-301.

\section{NOMENCLATURE}

\begin{tabular}{|c|c|}
\hline$A_{p}$ & superficial area of droplet \\
\hline$C_{D}$ & drag coefficient \\
\hline$c_{p}$ & specific heat of droplet \\
\hline$D_{\mathrm{p}}$ & actual diameter of droplet \\
\hline$d$ & diameter \\
\hline$d x$ & half of the length of cell in $\mathrm{x}$ direction \\
\hline$d y$ & half of the length of cell in y direction \\
\hline$d z$ & half of the length of cell in $\mathrm{z}$ direction \\
\hline E & energy \\
\hline$E_{i}$ & energy of phase $\mathrm{i}$ \\
\hline$E_{m}$ & total water collection coefficient \\
\hline $\mathbf{F}$ & body force \\
\hline $\mathbf{g}$ & acceleration of gravity \\
\hline$h$ & convective heat transfer coefficient \\
\hline$h_{f g}$ & latent heat of evaporation \\
\hline$k_{\text {eff }}$ & effective thermal conductivity \\
\hline$L$ & characteristic length of structure \\
\hline$M_{f}$ & liquid mass in cell \\
\hline$M_{p}$ & mass of droplet in discrete phase \\
\hline$m_{p}$ & mass of droplet \\
\hline$p$ & pressure \\
\hline $\operatorname{Re}$ & Reynolds number \\
\hline$R_{i}$ & $\begin{array}{l}\text { distance from the cell centre to the } \\
\text { particle centre }\end{array}$ \\
\hline $\mathbf{S}_{\mathrm{m}}$ & source term of momentum \\
\hline$S_{\rho}$ & source term \\
\hline$S_{t}$ & source term of energy \\
\hline$T_{f}$ & temperature of fluid \\
\hline$T_{i}$ & temperature of cell $\mathrm{i}$ \\
\hline
\end{tabular}

$\begin{array}{ll}T_{p} & \text { temperature of droplet } \\ T_{\infty} & \text { temperature of air around the droplet } \\ t & \text { time } \\ t^{*} & \begin{array}{l}\text { dimensionless time after } \\ \text { impingement } \\ \mathbf{U}_{p}\end{array} \\ \mathbf{u} & \text { velocity of single droplet } \\ V_{c e l l} & \text { air velocity around the droplet } \\ \alpha_{i} & \text { volume of cell } \\ \delta & \text { volume fraction of phase i } \\ \mu & \text { switch function } \\ \rho & \text { coefficient of dynamic viscosity } \\ \rho_{\mathrm{d}} & \text { density } \\ \rho_{1} & \text { density of droplet } \\ \rho_{i} & \text { density of liquid }\end{array}$

\section{ACKNOWLEDGMENTS}

This work is partially supported by the Natural Science Foundation of China (Grant No. 51706244) and National Science and Technology Specific Projects for Aero-engine and Gas Turbine (No. 2017-VIII-0003-0114). 